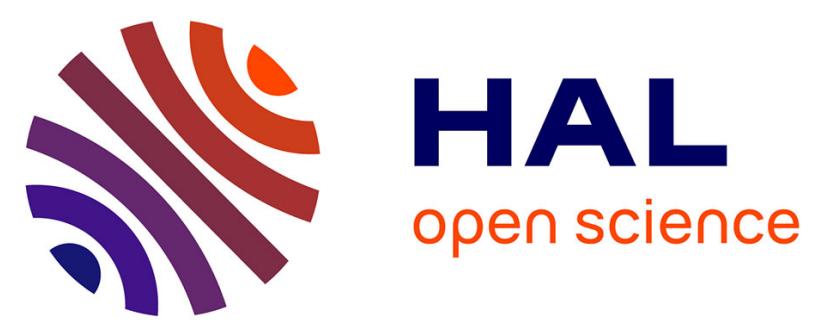

\title{
Transformations structurales dans les aciers inoxydables austeno-ferritiques: influence des éléments d'alliage
}

\author{
J. Gagnepain, F. Dupoiron, B. Bonnefois, Jérôme Charles
}

\section{To cite this version:}

J. Gagnepain, F. Dupoiron, B. Bonnefois, Jérôme Charles. Transformations structurales dans les aciers inoxydables austeno-ferritiques : influence des éléments d'alliage. Journal de Physique IV Proceedings, 1994, 04 (C3), pp.C3-75-C3-82. 10.1051/jp4:1994310 . jpa-00252507

\section{HAL Id: jpa-00252507 https://hal.science/jpa-00252507}

Submitted on 1 Jan 1994

HAL is a multi-disciplinary open access archive for the deposit and dissemination of scientific research documents, whether they are published or not. The documents may come from teaching and research institutions in France or abroad, or from public or private research centers.
L'archive ouverte pluridisciplinaire HAL, est destinée au dépôt et à la diffusion de documents scientifiques de niveau recherche, publiés ou non, émanant des établissements d'enseignement et de recherche français ou étrangers, des laboratoires publics ou privés. 


\title{
Transformations structurales dans les aciers inoxydables austeno-ferritiques : influence des éléments d'alliage
}

\author{
J.C. GAGNEPAIN, F. DUPOIRON, B. BONNEFOIS et J. CHARLES
}

Creusot-Loire Industrie, 56 Rue Clémenceau, BP. 56, 71202 Le Creusot cedex, France

\begin{abstract}
:
This paper describes the structural changes which occur during both isothermal and anisothermal heat treatments, then the roles of the individual alloying elements are discussed. In particular, nitrogen additions are shown to have a beneficial influence on the stability of the two phase structure during high temperature annealing $\left(1000-1300^{\circ} \mathrm{C}\right)$.
\end{abstract}

\section{1 - INTRODUCTION :}

Les aciers duplex doivent leur nom au fait qu'ils possèdent une structure biphasée, composée en proportions volumiques approximativement équivalentes d'austénite et de ferrite.

Les principaux avantages de ce type de nuance sont :

$>$ une limite d'élasticité élevée (460 MPa) de l'ordre de deux fois celle des nuances austénitiques standards ;

$>$ une résistance à la corrosion excellente (corrosion localisée et corrosion sous contrainte) ;

$>$ un coût raisonnable dû à leur basse teneur en nickel $(\sim 5 \%)$.

Ce papier présente les différentes transformations structurales affectant ce type de nuances ainsi que les effets des principaux éléments d'alliage. 


\section{2 - COMPOSITIONS CHIMIOUES :}

Table 1 : Composition chimique des nuances austéno-ferritiques ( \% massiques ) :

\begin{tabular}{|c|c|c|c|c|c|c|c|}
\hline NUANCE & UNS & Cr & Ni & Mo & $N$ & Cu & PREN \\
\hline UR 35N & S39230 & 23 & 4 & - & 0,10 & - & 25 \\
UR 45N & S39209 & 22 & 5,5 & 3 & 0,17 & - & 35 \\
UR 47N & $(S 39226)$ & 25 & 6,5 & 3 & 0,20 & - & 38 \\
UR 52N & $S 39255$ & 25 & 6,5 & 3 & 0,20 & 1,6 & 38 \\
UR 52N+ & $S 39255$ & 25 & 6,5 & 3,5 & 0,25 & 1,6 & 41 \\
& & & & & & & \\
\hline
\end{tabular}

La table 1 présente la composition chimique de divers aciers austéno ferritiques. Cette table donne également les valeurs correspondantes d'un indice de résistance à la corrosion par piqûres, le PREN = $\mathrm{Cr}+3,3 \mathrm{Mo}+16 \mathrm{~N}$. On distingue aujourd'hui trois principaux types de nuances austéno-ferritiques :

$>$ la nuance type $22 \% \mathrm{Cr}-5 \% \mathrm{Ni}-3 \% \mathrm{Mo}-0,17 \% \mathrm{~N}$ (UNS 39209, UR45N), qui peut être considérée comme la nuance duplex "standard". Sa résistance à la corrosion est intermédiaire entre celle d'un acier austénitique de base type $316 \mathrm{~L}$ et celle d'aciers super austénitiques à 5-6\% Mo ;

$>$ les nuances dites "super duplex" caractérisées par un indice de résistance à la piqûre PREN > 40 (UNS 39255 UR52N+) qui contiennent approximativement $25 \% \mathrm{Cr}-6,5 \% \mathrm{Ni}-3,7 \% \mathrm{Mo}$ et $0,25 \% \mathrm{~N}$ avec ajouts de cuivre ou tungstène. Ces nuances présentent des caractéristiques mécaniques élevées et une résistance à la corrosion dans des environnements extrêmement agressifs (milieux chlorurés acides), leur résistance à la corrosion étant équivalente à celle des aciers super austé nitiques contenant 5-6\% Mo ;

$>$ la nuance type $23 \% \mathrm{Cr}-4 \% \mathrm{Ni}-0,10 \% \mathrm{~N}$ sans additions de molybdène (UNS 39230, UR35N) qui peut remplacer dans certaines conditions des nuances type 304 et/ou 316 .

\section{3 - MICROSTRUCTURE DES ACIERS AUSTENO-FERRITIOUES}

\section{1 - Microstructure à l'état hypertrempé}

La figure 1 présente une section schématique du diagramme $\mathrm{Fe}-\mathrm{Ni}-\mathrm{Cr}$, pour une teneur en fer de $68 \%$. 


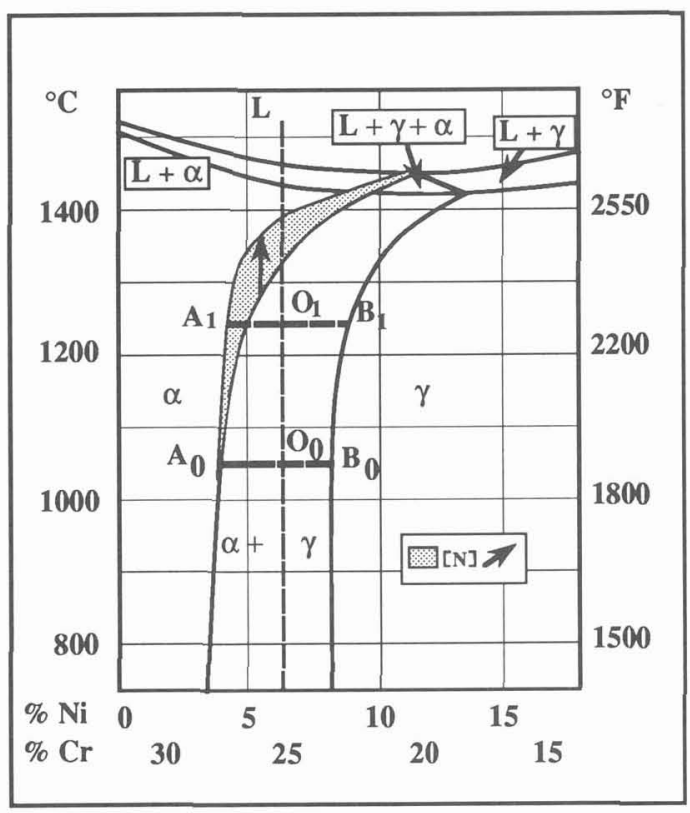

Fig. 1 : Diagramme pseudo binaire $\mathrm{Cr}-\mathrm{Ni}-68 \mathrm{Fe}$

Les aciers duplex se solidifient dans le domaine ferritique et c'est au cours du refroidissement qu'une partie de la ferrite se transforme en austénite.

Les aciers duplex produits à l'heure actuelle ont des proportions approximativement équivalentes d'austénite et ferrite, ceci étant obtenu par la maîtrise des conditions de transformations à chaud, du traitement d'hypertrempe et bien sûr de la composition chimique de l'acier.

Un exemple de structure obtenue sur tôle à l'état hypertrempé est présenté figure 2.

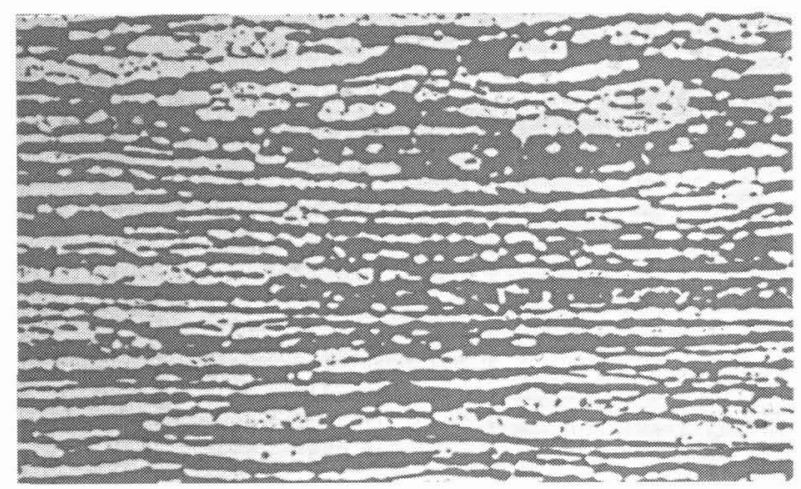

Figure 2 : Microstructure à l'état hypertrempé.

En pratique, la température de traitement thermique doit être choisie aussi basse que possible, mais suffisante pour remettre en solution toutes les précipitations. Les proportions relatives de ferrite et d'austénite sont alors essentiellement déterminées par la compositon chimique de la nuance, particulièrement par l'équilibrage entre éléments stabilisateurs de la ferrite $(\mathrm{Cr}, \mathrm{Mo}, \mathrm{Si}$...) et éléments stabilisateurs de l'austénite ( $\mathrm{Ni}, \mathrm{N}, \mathrm{C}$ ). La qualité et la précision de l'élaboration sont les seules garanties d'un contrôle de la microstructure. 


\section{2 - Stabilité de la structure avec la température}

La figure 3 illustre bien l'action fortement gammagène de l'azote.

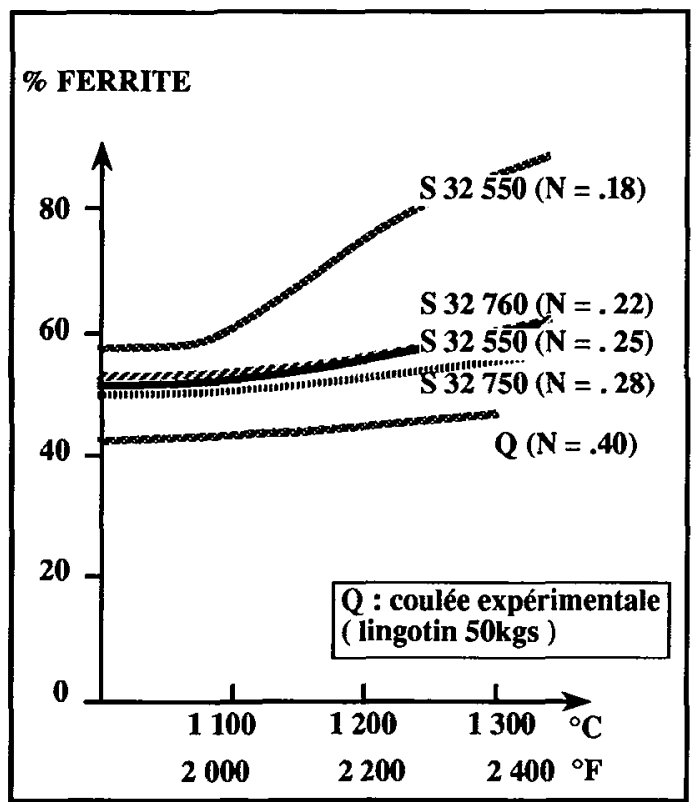

Fig 3 : Variation de la teneur en ferrite avec la température ( duplex à $25 \% \mathrm{Cr}$ )

De plus l'augmentation de la teneur en ferrite avec la température est fortement ralentie par les additions d'azote, cette stabilisation de la structure par l'azote est d'un grand intérêt dans le cas des ZAT de soudage.

\section{3 - Coefficients de nartage}

La table 2 présente les valeurs des coefficients de partage des divers éléments d'alliage entre ferrite et austénite (déterminé par analyse microsonde).

Table 2 : Coefficients de partage $([\alpha]) /([\gamma])$ entre ferrite et austénite.

\begin{tabular}{|c|c|c|c|c|c|c|c|c|c|}
\hline NUANCE & $\mathrm{T}\left({ }^{\circ} \mathrm{C}\right)$ & $\mathrm{Cr}$ & $\mathrm{Ni}$ & $\mathrm{Mo}$ & $\mathrm{N}$ & $\mathrm{Si}$ & $\mathrm{Cu}$ & $\mathrm{Mn}$ & $\mathrm{P}$ \\
\hline UR 35N & 960 & 1,19 & 0,61 & 1,65 & - & 1,16 & 0,68 & 0,89 & 2,38 \\
UR 45N & 1050 & 1,22 & 0,56 & 1,66 & $0,2^{*}$ & 1,22 & 0,63 & 0,85 & 2,31 \\
UR 52N & 1040 & 1,15 & 0,65 & 1,6 & - & 1,19 & 0,69 & 0,87 & 2,9 \\
UR 52N+ & 1080 & 1,12 & 0,66 & 1,60 & 0,09 & 1,18 & 0,71 & 0,87 & - \\
\hline
\end{tabular}

$* \% \mathrm{~N}=0,05$ dans $\alpha, 0,25$ dans $\gamma \quad * * \% \mathrm{~N}=0,035$ dans $\alpha, 0,40$ dans $\gamma$

Plusieurs points importants sont à souligner : 
a) La ferrite est enrichie en éléments alphagènes avec dans l'ordre $\mathrm{P}>\mathrm{W}>\mathrm{Mo}>\mathrm{Cr} \sim \mathrm{Si}$.

b) L'austénite est enrichie en éléments gammagènes, avec dans l'ordre $\mathrm{N} \gg \mathrm{Ni}>\mathrm{Cu}>\mathrm{Mn}$.

c) Excepté pour l'azote, les coefficients de partage varient très peu d'une nuance à une autre.

Ceci s'explique par le fait qu'hormis pour l'azote, dans les domaines de concentrations et températures de traitement concernés, les limites de solubilité pour les autres éléments n'ont pas été atteintes.

d) Le coefficient de l'azote semble quant à lui varier fortement avec sa teneur totale dans la nuance. En fait la ferrite est rapidement saturée, et l'excès d'azote passe en solution dans l'austénite. La limite de solubilité de l'azote dans la ferrite est de l'ordre de $0,03-0,05 \%$, ce pour les compositions et températures de traitement thermique étudiées.

En pratique toute augmentation de la teneur en azote de l'acier duplex mènera donc à un enrichissement marqué en cet élément de l'austénite, sans en changer le niveau dans la ferrite ce tant que la température de traitement n'est pas modifiée.

\section{4 - TRANSFORMATIONS STRUCTURALES PRODUTES PAR LES TRAITEMENTS THERMIOUES}

De nombreuses transformations microstructurales peuvent affecter les aciers austéno-ferritiques durant les traitements thermiques isothermes ou anisothermes. Beaucoup de ces transformations concernent la phase ferritique, ceci s'expliquant par le fait que les vitesses de diffusion des éléments d'alliage dans cette phase sont de l'ordre de 100 fois plus grande que dans l'austénite. Ceci est principalement la conséquence de la structure cubique centrée moins compacte de la ferrite [1].

De plus, la ferrite est enrichie en $\mathrm{Cr}$ et Mo qui sont connus pour favoriser les précipitations de phases intermétalliques. Enfin la solubilité dans la ferrite des éléments $C, N$, W, et Cu chute fortement avec une température décroissante, augmentant la probabilité de précipitations durant les traitements thermiques.

\section{1 - Transformations lors de maintiens entre 600 et $1000^{\circ} \mathrm{C}$}

De nombreux changements microstructuraux peuvent apparaître dans les aciers duplex après maintiens entre $600^{\circ} \mathrm{C}$ et $1000^{\circ} \mathrm{C}$. On peut citer les phases intermétalliques $\sigma, \chi, \mathrm{R}$, les carbures et nitrures, l'austénite secondaire [2].

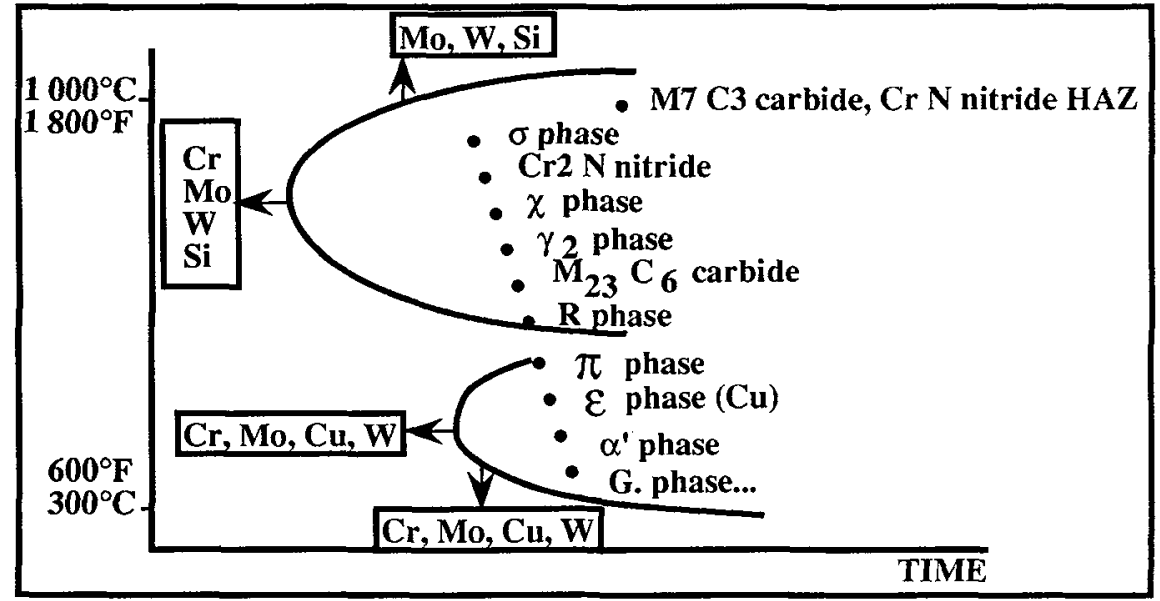

Figure 4 : Précipitations possibles dans les aciers inoxydables duplex 


\subsection{1 - Phases intermétalliques :}

La plus communémEnt rencontrée est la phase $\sigma$ qui est connue pour son effet particulièrement désastreux sur les caractéristiques mécaniques. Sa composition chimique est typiquement proche de $\mathrm{Fe}$ $30 \% \mathrm{Cr}-4 \% \mathrm{Ni}-4$ à $7 \% \mathrm{Mo}$. Dans la pratique on la confond souvent avec la phase $\chi(\mathrm{Fe}-25 \% \mathrm{Cr}$ $3 \% \mathrm{Ni}-14 \% \mathrm{Mo}$ ).

Ces phases intermétalliques sont plus stables à haute température en présence de W, Mo, Si obligeant la réalisation de traitements d'hypertrempe à températures plus élevées.

Leur cinétique de précipitations est également accélérée par des additions croissantes de $\mathrm{W}, \mathrm{Mo}, \mathrm{Cr}$ et Si.

\subsection{2 - Carbures et nitrures :}

- Les premiers aciers duplex produits (UR50 par exemple) étaient caractérisés par de basses teneurs en azote et des niveaux de carbone de l'ordre de $0,03 \%$. Les premiers précipités à se former étaient alors des carbures de type $\mathrm{M}_{7} \mathrm{C}_{3}$ aux environs de $900-1050^{\circ} \mathrm{C}$ et de type $\mathrm{M}_{23} \mathrm{C}_{6}$ à des températures plus basses. De par leurs natures discontinues, de telles précipitations n'affectent pas forcément les propriétés mécaniques ou la résistance à la corrosion.

Les nuances duplex à l'heure actuelle ont des teneurs en carbone souvent inférieures à $0,015 \%$ tandis que leur teneur en azote est optimisée en fonction des teneurs en $\mathrm{Cr}$ et Mo puisque sa solubilité est augmentée par ces deux éléments.

- Les précipités de type $\mathrm{Cr}_{2} \mathrm{~N}$ peuvent apparaître dans le domaine $700-900^{\circ} \mathrm{C}$.

Il faut souligner que leur cinétique de précipitation dépend fortement des conditions de traitement thermique. Une température de traitement élevée $\left(1200-1300^{\circ} \mathrm{C}\right)$ peut mener à une dissolution plus importante des interstitiels $\mathrm{C}, \mathrm{N}$ dans la ferrite. En conséquence une précipitation abondante de nitrures peut apparaître durant le refroidissement. Cette précipitation de nitrures peut affecter les propriétés d'usage des aciers austéno-ferritiques, particulièrement la résistance à la corrosion, à cause de l'appauvrissement local en chrome.

\section{2 - Transformations à basse température $\left(<550^{\circ} \mathrm{C}\right)$}

Comme pour les aciers ferritiques à haute teneur en chrome, la ferrite des aciers duplex peut être durcie par traitement entre 300 et $550^{\circ} \mathrm{C}$. Ce durcissement peut être causé par différentes transformations : formation de phase $\alpha^{\prime}$, précipitations de carbures, nitrures, précipités riches en cuivre.

Le durcissement de la ferrite est généralement attribué à la formation de phase $\alpha^{\prime}$ par décomposition spinodale. La ferrite se sépare spontanément en zones alternativement enrichies ou appauvries en chrome. A des températures un peu plus élevées, il n'est pas exclu que la transformation se fasse par germination et croissance. La formation de cette phase est facilitée par de hautes teneurs en $\mathrm{Cr}$ et en éléments interstitiels et, à un degré moindre, par des additions de titane, aluminium et cuivre. Le rôle du molybdène reste incertain.

Des maintiens prolongés à $400^{\circ} \mathrm{C}$ peuvent mener également à la formation de carbures et nitrures qui contribuent au durcissement de la ferrite par leur faible taille.

Enfin, pour des nuances contenant du cuivre et/ou du tungstène, d'autres mécanismes de durcissement apparaissent. Dans le cas du cuivre, la sursaturation de la ferrite due à la décroissance de sa solubilité aux basses températures, se traduit par la précipitation de particules extrêmement fines enrichies en cet élément et étend le domaine de durcissement vers les basses températures $[1,3]$. 


\section{3 - Transformations en refroidissement continu}

Pour des traitements industriels, il est important de se souvenir, qu'aux températures élevées proches de la température de remise en solution, la nucléation des précipités est lente et leur croissance est rapide, alors que c'est l'inverse aux plus basses températures au voisinage du nez de précipitations. Il peut être ainsi difficile d'éviter la précipitation de phases durant le réchauffage de produits épais et il est alors nécessaire d'accomplir le traitement thermique à une température suffisamment haute pour remettre en solution les phases formées lors du chauffage.

Au contraire, durant le refroidissement, la faible vitesse de nucléation à haute température et la lente cinétique de croissance à plus basse température font qu'il est relativement facile d'éviter la formation de phases.

La figure 5 montre l'exemple d'un diagramme de refroidissement continu pour la nuance UR52N+. Après hypertrempe à $1080^{\circ} \mathrm{C}$, des vitesses aussi lentes que $2000^{\circ} \mathrm{C} / \mathrm{h}$ sont réalisables sans précipitations. Des vitesses de refroidissement plus rapides sont recommandées en cas de traitement à plus hautes températures puisque, comme nous l'avons vu, le risque de précipitation de nitrures est accru.

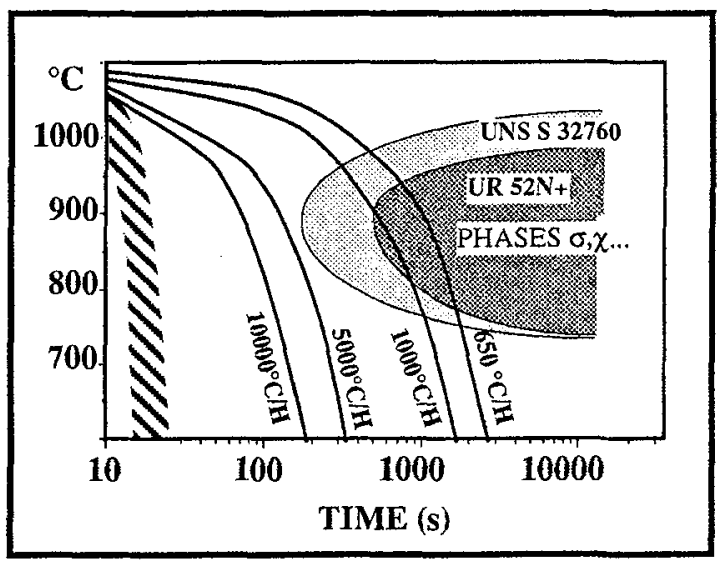

Figure 5 : Diagramme de refroidissement continu (chauffage à $1080^{\circ} \mathrm{C}$ ) Vitesses de refroidissement typiques d'une ZAT

\section{4 - Variations des proportions d'austénite et mornhologie}

Les proportions respectives d'austénite et ferrite dépendent de la température. Durant le cycle de refroidissements un taux croissant de ferrite se transforme en austénite.

En cas de refroidissement lent, ce phénomène se produit par déplacement des joints de grain ferrite/austénite.

D'autres morphologies (aciculaires) d'austénite secondaire peuvent apparaître. Ces précipitations de carbures nitrures et/ou phases intermétalliques peuvent mener à la formation d'austénite due à des appauvrissements locaux en $\mathrm{Cr}$ et $\mathrm{Mo}$.

La formation d'austénite secondaire $\gamma_{2}$, en particulier dans les zones affectées thermiquement, est marquée par une variation locale de la composition chimique, dégradant la résistance à la corrosion (cas en particulier des ZAT de soudage) et peut être le site privilégié de la nucléation de nitrures ou composés intermétalliques $[1,2]$. 


\section{5 - CONCLUSION :}

Cet article a présenté les principales transformations microstructurales pouvant affecter les aciers austéno-ferritiques, transformation affectant particulièrement la phase ferritique. Un traitement thermique d'hypertrempe bien maîtrisé permet de s'affranchir de la présence de précipitations souvent néfastes pour les propriétés mécaniques et/ou la résistance à la corrosion.

$\mathrm{Du}$ fait des domaines de précipitations caractérisant ce type de nuances leurs applications potentielles sont souvent limitées à des températures inférieures à $300^{\circ} \mathrm{C}$.

Enfin, rappelons que la connaissance des effets des éléments d'alliage (en particulier de l'azote) sur la cinétique de ces précipitations a permis de développer de nouveaux aciers austéno-ferritiques dits "super duplex" caractérisés par un PREN $\geq 40$, et offrant d'excellentes propriétés de résistance à la corrosion.

\section{REFERENCES :}

[1] CHARLES J., Super duplex stainless steels : structure and properties, Duplex Stainless Steels'91, Beaune, Proceedings Vol 1 p 3-48.

[2] JOSEPHSON B., NISSON J.O., WILSON A, phase transformations duplex steels and the relations between continuous cooling and isothermal heat treatment, Duplex Stainless Steels'91, Beaune, Proceedings, Vol. 1 p 67 - 78.

[3] CHARLES J., Super duplex stainless steels : structure and properties. Duplex stainless Steels'91, Beaune, Proceedings, Vol. 1, p 151 - 168. 